\title{
Imaging Characteristics and Management of Infected Hepatic Hemangioma: Case-in Discussion
}

\author{
Karolin Ginting ${ }^{1}$ Anisha Tailor ${ }^{2}$ Timothy Braverman ${ }^{3}$ \\ ${ }^{1}$ Department of General Surgery, The Jewish Hospital, Cincinnati, \\ Ohio, United States \\ 2Department of Internal Medicine, McLaren Macomb Hospital, \\ Mount Clemens, Michigan, United States \\ ${ }^{3}$ Department of Pathology, The Jewish Hospital, Cincinnati, Ohio, \\ United States \\ ${ }^{4}$ Department of Radiology, The Jewish Hospital, Cincinnati, Ohio, \\ United States
}

Ajay Agarwal $^{4} \quad$ Shyam Allamaneni ${ }^{1}$

\author{
Address for correspondence Karolin Ginting, MD, Department \\ of General Surgery, The Jewish Hospital, 4777 E. Galbraith Road, \\ Cincinnati, OH 45236, United States (e-mail: kginting@mercy.com).
}

J Gastrointestinal Abdominal Radiol ISGAR 2021;4:236-239.

\begin{abstract}
Keywords

- hemangioma

- liver abscess

- drainage

Hepatic hemangiomas are the most common type of benign liver tumors. We present a case of an infected hepatic hemangioma, which posed a diagnostic challenge. A 43-year-old female presented with right upper quadrant pain after blunt force trauma and intentional weight loss. CT and MRI were done, showing a posterior right lobe liver mass. Imaging characteristics were thought to be suggestive of metastatic disease, with a differential diagnosis of abscess. Drainage and biopsy of the mass revealed Streptococcus sp. infection, and pathology showed a cavernous hemangioma with inflammation. Patients presenting with systemic symptoms and an indeterminate liver mass on imaging should be evaluated for infected hepatic hemangiomas. Biopsy may be needed to rule out cancer. Management may include surgical resection; however, antibiotics and percutaneous drainage can suffice, as in this case.
\end{abstract}

\section{Introduction}

Hepatic hemangiomas are the most common type of benign tumors of the liver. They are typically discovered as an incidental finding on cross-sectional imaging performed for other reasons. ${ }^{1}$ The etiology of hepatic hemangiomas is thought to be due to atypical enlargement of abnormal hepatic vasculature or congenital vascular ectasia. Angiogenic factors and hormonal status are known factors in its pathogenesis. Females have a strong predisposition to this tumor, especially those who are on oral contraceptives, steroid therapy, or who are pregnant.

These lesions are typically small, benign and, rarely, cause complications. Pregnancy, steroid use and hormonal therapy, including oral contraceptives, are associated with growth of this tumor. Cessation of hormonal exposure will usually result in shrinkage of the tumor size. When present, hepatic hemangiomas may cause chronic pain (due to Glisson's capsule irritation) or acute pain (due to rupture). Other symptoms may include abdominal fullness due to the compressive effect on the gastrointestinal tract, in which case surgical resection is recommended. Prophylactic resection of large or peripherally located hemangiomas are also indicated in individuals who are at high risk of traumatic rupture. ${ }^{2,3}$ There are multiple case reports regarding management of complicated giant hemangiomas and infected hepatic hemangiomas. ${ }^{4,5}$ We present a case of infected hepatic hemangioma associated with trauma, where initial imaging was concerning for metastasis. published online June 11, 2021
DOI https://doi.org/

$10.1055 / \mathrm{s}-0041-1728982$ ISSN 2581-9933 (c) 2021. Indian Society of Gastrointestinal and Abdominal Radiology. This is an open access article published by Thieme under the terms of the Creative Commons Attribution-NonDerivative-NonCommercial-License, permitting copying and reproduction so long as the original work is given appropriate credit. Contents may not be used for commercial purposes, or adapted, remixed, transformed or built upon. (https://creativecommons.org/licenses/by-nc-nd/4.0/).

Thieme Medical and Scientific Publishers Pvt. Ltd. A-12, 2nd Floor, Sector 2, Noida-201301 UP, India 


\section{Case Presentation}

A 43-year-old female presented to the emergency department with a chief complaint of worsening right upper quadrant abdominal pain, following a blunt trauma from a go-cart collision 2 days prior. A contrast-enhanced CT revealed a hypodense (with average density of 38 Hounsfield units) subcapsular lesion in the posterior right lobe of the liver, measuring $50 \times 47 \mathrm{~mm}$. This mass had no rim enhancement (-Fig. 1). Additional tiny hypodense lesions were noted in the right lobe and the dome of the liver. Notably, an indeterminate left adrenal gland nodule $(12 \mathrm{~mm})$ was noted.

Follow-up MRI was completed the following day after CT study. This showed hypointense subscapular posterior right lobe liver mass on T1-weighted images (T1WI) (-Fig. 2A). This mass was heterogeneously hyperintense on T2-weighted images (T2WI) (-Fig. 2B), with associated gradual enhancement and diffusion restrictions ( - Fig. 3 ). There was gradual central enhancement with peripheral nonenhancing rim, which persisted during the hepatocyte phase (-Fig. 4). Additional small liver lesions were consistent with cysts. The left adrenal gland nodule that was found on CT scan was consistent with a benign adenoma.

Given these imaging findings, malignancy needed to be excluded. Upon further questioning, patient gave history of weight loss, but she sated it was intentional. Thus, a percutaneous biopsy was obtained to rule out malignancy, which was performed 2 days after MRI study. Four milliliters of purulent fluid was aspirated on CT-guided biopsy; thus, a drainage catheter was placed. This fluid collection grew Streptococcus sp., and the patient was treated with antibiotics.
A core needle biopsy was also performed, and subsequent histologic evaluation favored a diagnosis of cavernous hemangioma with infarction and inflammation (-Fig. 5). The patient was discharged after an 8-day hospital course, with a percutaneous drain and intravenous (IV) antibiotic treatment for an additional 4 weeks. A week following discharge, an abscessogram study was completed to evaluate for possible fistula formation to adjacent structure. This showed filling of the abscess cavity, without contrast extravasation outside of the cavity (-Fig. 6). The drain was then removed during outpatient follow-up a week later. The patient was doing well at her six-month follow-up.

\section{Discussion}

Hepatic hemangiomas are the most common benign hepatic lesions, which can typically be diagnosed with imaging studies. ${ }^{6}$ The classic presentation of a hemangioma on contrast-enhanced CT is as a smooth, well-demarcated mass with peripheral nodular enhancement, followed by a filling-in pattern during the late phase. The lesion remains isodense or hyperdense on the delayed phase imaging. ${ }^{6,7}$ On sonographic study, the majority of hemangiomas present as homogeneous, hyperechoic masses with posterior enhancement. ${ }^{1}$ MRI is considered the gold standard modality in the diagnosis of hepatic hemangiomas. ${ }^{8}$ It is characterized by a hyperintense mass on T2WI, with signal intensity between cerebrospinal fluid and the spleen, and hypointense on T1WI. Larger hemangiomas may have an area of nonenhancement on T1WI. ${ }^{9,10}$ The sensitivity and specificity of an MRI study is increased to $100 \%$ with contrast enhancement. ${ }^{11}$
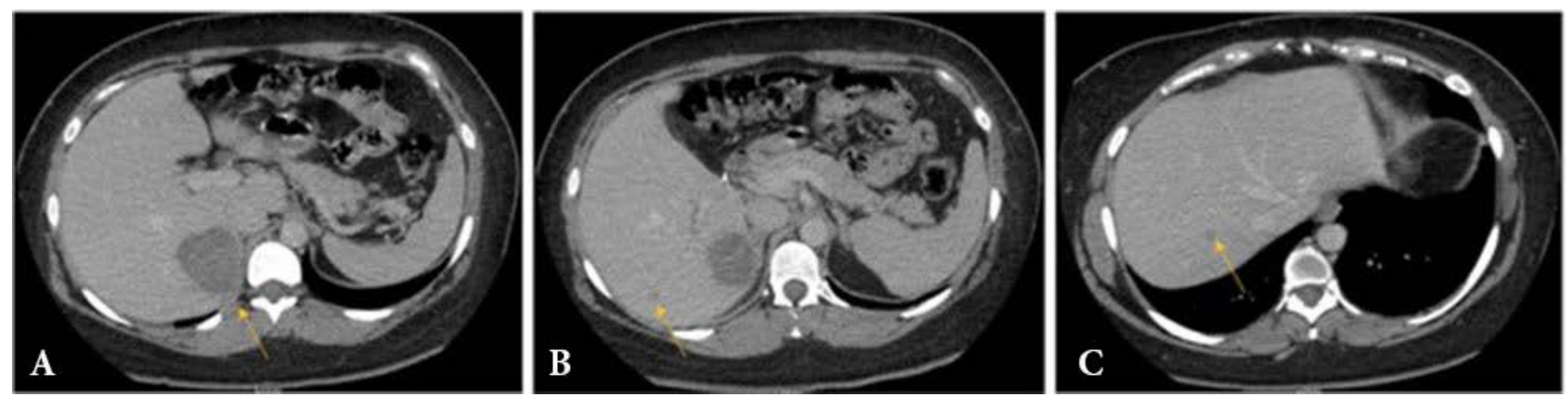

Fig. 1 Contrast-enhanced computed tomography axial image demonstrate a hypoattenuating lesion in posterior right hepatic lobe (segment 6) (A). Additional tiny hypodense lesions were noted in the right lobe and the dome of the liver (B, C).
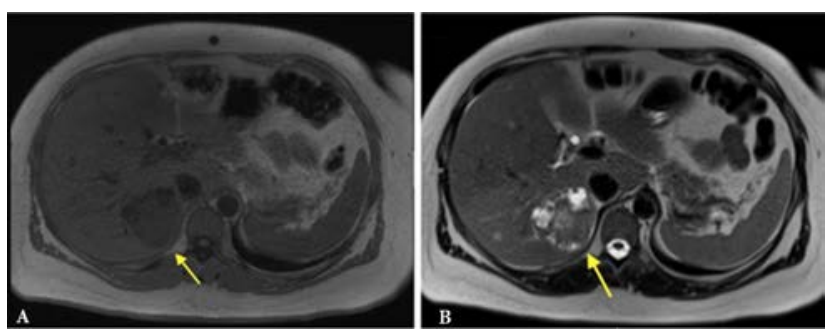

Fig. 2 T1-weighted MRI axial image demonstrate a hypointense lesion in the posterior right hepatic lobe (A). T2-weighted MRI axial image demonstrate a heterogeneously hyperintense lesion in the posterior right lobe (B).
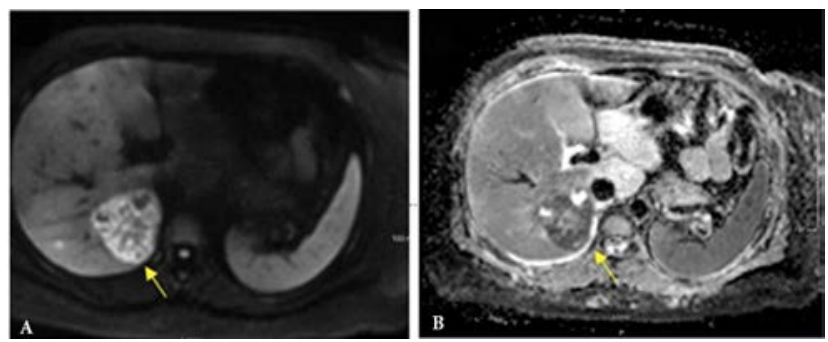

Fig. 3 Diffusion-weighted-image (A) with apparent diffusion coefficient map showed diffusion restriction (B). 

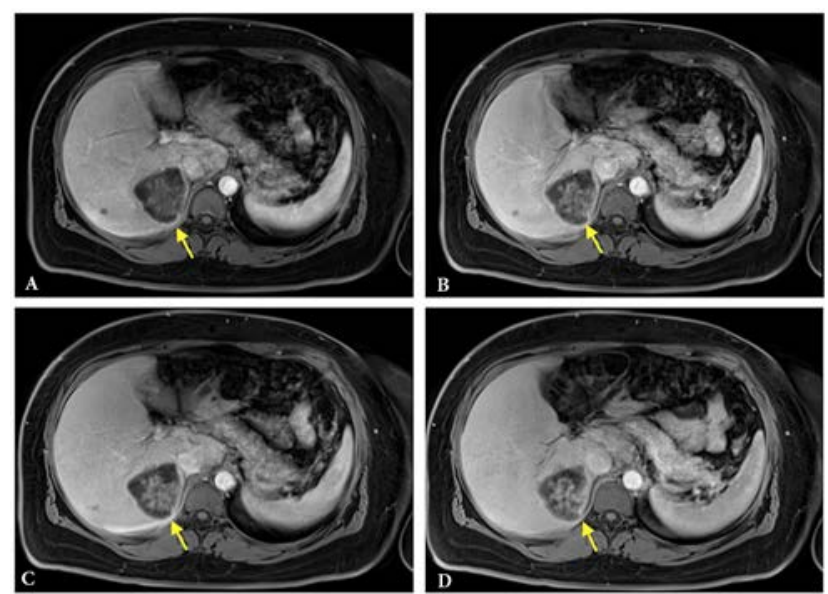

Fig. 4 (A-D) Contrast-enhanced MRI axial images demonstrate progressive central enhancement with minimal peripheral rim enhancement.

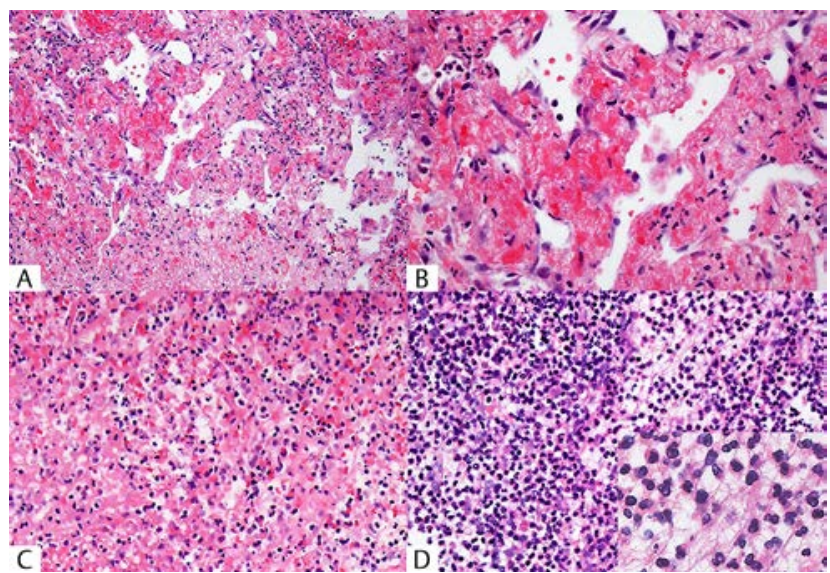

Fig. 5 The upper half of the photomicrograph highlights viable if congested hemangioma at 20x (A) and 40x (B) magnification. The lower half highlights the infected portion at $40 \mathrm{x}$ magnification including mild-to-moderate (C), and florid, with electronically enhanced inset of neutrophils (D).

While MRI is highly accurate in the diagnosis of typical hemangiomas, atypical features should be explored further, as they broaden the differential and increase the challenge of diagnosing a hepatic hemangioma. Only $10 \%$ of hemangioma cases will show multiple lesions and this should prompt further evaluation. ${ }^{12}$ As noted in our patient, CT imaging showed a hypodense lesion in the posterior right lobe of the liver, which measured $50 \times 47 \mathrm{~mm}$, with no rim enhancement. Additional hypodense lesions were noted in the right lobe and the dome of the liver, which was concerning for metastasis. This led to further workup with MRI, where the mass was also found to be heterogeneously hyperintense on an MR T2WI, with a nonenhancing peripheral rim. Multiphase CT scan was not done in lieu of MRI study.

Heterogeneity findings in hepatic hemangiomas can be found in large and giant hemangiomas (size greater than $4 \mathrm{~cm}$ ) and can be due to thrombosis, fibrosis, calcification, hemorrhaging necrosis or obliteration of cavernous sinuses. ${ }^{1,13,14}$ The differential diagnosis when atypical features are present must be all-encompassing, including focal nodular

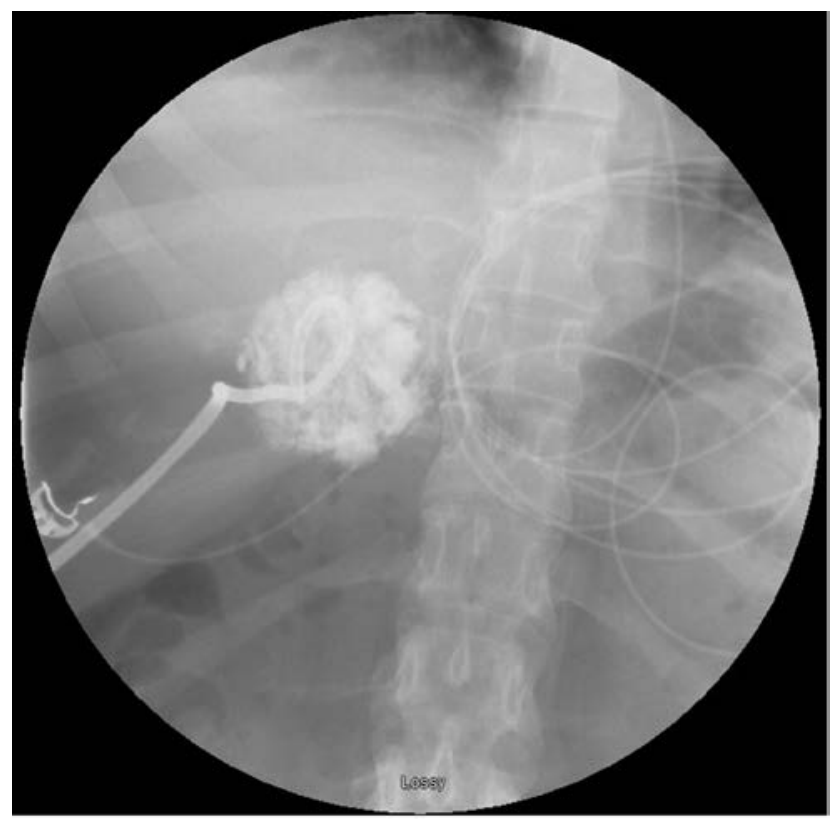

Fig. 6 Contrast injection into the percutaneous drain showed abscess cavity without extravasation outside of the abscess cavity such as to the biliary system or any other hepatic structure.

hyperplasia when scarring is seen, primary hepatic tumors, and metastases, among many others. Hemangiomas with inflammatory processes are very rare and associated with giant hemangiomas. Previous case reports describe these findings to show typical giant hemangioma characteristics. ${ }^{13}$

Although the MRI study of this patient showed heterogeneously $\mathrm{T} 2$ hyperintense and $\mathrm{T} 1$ hypointense, associated gradual enhancement with diffusion restrictions favored metastasis rather than hemangioma (-Figs. 2 and 3 ). Differential diagnosis of metastasis was also supported by symptoms of weight loss endorsed by the patient. Other differential diagnoses included hepatocellular carcinoma and hepatic adenoma. The patient was recommended CT-guided needle biopsy. This was performed and purulent drainage was noted at the time of biopsy, which led to placement of a percutaneous drain for source control. Fluid culture and pathology study showed a final diagnosis of infected hemangioma.

Giant hemangioma is usually diagnosed incidentally during imaging study for other disease processes. In symptomatic patients, such as pain, bloating, and discomfort, surgical resection or enucleation can be offered. ${ }^{3}$ Other indications for surgical resection are uncertainty in diagnosis and enlarging size. Alternatively, nonsurgical management should also be discussed and may result in decreasing in size and symptoms' resolution. Imaging-guided percutaneous aspiration of hepatic cavernous hemangioma has been shown to be safe and useful in diagnosis. ${ }^{15}$ However, caution should be taken when performing this procedure, given the high vascularity of the tumor. ${ }^{16}$ It is important to obtain safe route and use small needle to initiate the procedure. In our case, percutaneous drain placement after biopsy and fluid aspiration allowed patient to avoid partial liver resection. 
In conclusion, we documented a case of infected hepatic hemangioma, which was found in a patient who presented with right upper quadrant pain secondary to trauma. This diagnosis should be considered when a patient presents with systemic inflammatory symptoms, and subsequent imaging findings of a hepatic mass are inconclusive. Usually, biopsy is needed to rule out malignancy like in our patient. In addition, this case demonstrates that nonsurgical treatment with antibiotics and percutaneous drainage may be sufficient to treat such patients.

\section{Conflict of Interest}

None declared.

\section{References}

1 Nelson RC, Chezmar JL. Diagnostic approach to hepatic hemangiomas. Radiology 1990;176(1):11-13

2 Abdel Wahab M, El Nakeeb A, Ali MA, et al. Surgical management of giant hepatic hemangioma: single center's experience with 144 patients. J Gastrointest Surg 2018;22(5):849-858

3 Pol B, Disdier P, Le Treut YP, Campan P, Hardwigsen J, Weiller PJ. Inflammatory process complicating giant hemangioma of the liver: report of three cases. Liver Transpl Surg 1998;4(3):204-207

4 Pinkernelle J, Neumann U, Hänninen EL. An unusual cause of epigastric pain: infected giant liver hemangioma. Acta Radiol 2007;48(2):142-144

5 Hoekstra LT, Bieze M, Erdogan D. Roelofs JJ, Beuers UH, van Gulik TM. Management of giant liver hemangiomas: an update. Expert Rev Gastroenterol Hepatol 2013;7(3):263-268

6 Klotz T, Montoriol P-F, Da Ines D, Petitcolin V, JoubertZakeyh J, Garcier J-M. Hepatic haemangioma: common and uncommon imaging features. Diagn Interv Imaging 2013;94(9): 849-859

7 Hanafusa K, Ohashi I, Himeno Y, Suzuki S, Shibuya H. Hepatic hemangioma: findings with two-phase CT. Radiology 1995;196(2):465-469

8 Seitz K, Bernatik T, Strobel D, et al. Contrast-enhanced ultrasound (CEUS) for the characterization of focal liver lesions in clinical practice (DEGUM Multicenter Trial): CEUS vs. MRI-a prospective comparison in 269 patients. Ultraschall Med 2010;31(5):492-499

9 Kania L, Guglielmo F, Mitchell D. Interpreting body MRI cases: classic findings in abdominal MRI. Abdom Radiol (NY) 2018;43(10):2790-2808

10 Mamone G, Miraglia R. The "light bulb sign" in liver hemangioma. Abdom Radiol (NY) 2019;44(6):2327-2328

11 Soussan M, Aubé C, Bahrami S, Boursier J, Valla DC, Vilgrain V. Incidental focal solid liver lesions: diagnostic performance of contrast-enhanced ultrasound and MR imaging. Eur Radiol 2010;20(7):1715-1725

12 Vásquez Montoya JD, Molinares B, Vásquez Trespalacios EM, García V, Pérez Cadavid JC. Atypical hepatic haemangiomas. BJR Case Rep 2017;4(1):20170029

13 Venkatesh SK, Chandan V, Roberts LR. Liver masses: a clinical, radiological and pathological perspective. Clin Gastroenterol Hepatol Off Clin Pract J Am Gastroenterol Assoc 2014;12(9): 1414-1429

14 Mamone G, Di Piazza A, Carollo V, et al. Imaging of hepatic hemangioma: from A to Z. Abdom Radiol (NY) 2020;45(3): 672-691

15 Nakaizumi A, Iishi H, Yamamoto R, et al. Diagnosis of hepatic cavernous hemangioma by fine needle aspiration biopsy under ultrasonic guidance. Gastrointest Radiol 1990;15(1):39-42

16 Vilgrain V, Boulos L, Vullierme M-P, Denys A, Terris B, Menu Y. Imaging of atypical hemangiomas of the liver with pathologic correlation. Radiographics 2000;20(2):379-397 